\title{
Rehabilitation of partially edentulous arch using semi precision attachment: an aesthetic approach
}

\author{
${ }^{1}$ Ainy Fitri Nuriyanto, ${ }^{2}$ Sherman Salim, ${ }^{2}$ Michael Josef K. K. \\ ${ }^{1}$ Resident of Prosthodontics \\ 2Department of Prosthodontics \\ Faculty of Dental Medicine Universitas Airlangga \\ Surabaya, Indonesia \\ Corresponding author: Michael Josef K.K., Email: josef_310563@yahoo.com
}

\begin{abstract}
The restoration of normal function and esthetic appearance with a dental prosthesis is a major challenge in the rehabilitation of patients who have lost their teeth. In such situation, a fixed removable prosthesis allows favorable biomechanical stress distribution along with restoration of esthetics, phonetics, comfort, hygieneand better postoperative care and maintenance. Prime function of attachment retained partial denture is to distribute the masticatory forces to the wide area thereby reducing the damage to the abutments, soft tissues and bony ridges in addition to improved esthetics and proprioceptive responses. In this case series patient's esthetic and functional requirements were fulfilled with attachment retained cast partial denture using semi-precision attachments
\end{abstract}

Keywords: semi-precision attachment, claspless, esthetic approach

\section{INTRODUCTION}

Precision attachments open a new horizon of possibilities in prosthodontic rehabilitation. ${ }^{1}$ From patient's perspective, retention is one of the important factors for its acceptability. ${ }^{2}$ Use of attachment in providing retention to removable prosthesis is an old treatmentmodality with better success. It highly improves the comfort, aesthetic, function, and patient satisfaction. ${ }^{3}$ Precisionattachment is an interlocking device, one component of which is fixed to an abutmentand the otheris integrated intoa removable partial denture to stabilize and/or to retain it. ${ }^{3}$

Thereare two types of attachments, namelyprecision attachment and semi-precision attachment. A precision attachment is fabricated from milled alloys and tolerances are within $0.01 \mathrm{~mm}$. They are generally intracoronal and non-resilient. Their advantages include consistent quality, controlledwear and easier repair. They have standard parts which are interchangeable. ${ }^{1}$

A semi-precision attachment is fabricated by the direct casting of plastic, wax, or refractory patterns. They are considered "semi-precision" since in their fabrication they are subject to inconsistent water/ powder ratios, burn out temperatures and othervariables. The resulting components therefore, vary to a small degree. They are less costly, easy to fabricate and may be cast in alloy. They are generally extra coronal and resilient. ${ }^{1}$

Based on location, the semi precision attachments are divided into ${ }^{4} 1$ )intracoronal attachments, that were placed within the contours of the crown form. The advantage ofan intracoronal attachment is that the occlusal forces exerted upon the abut- ment tooth are applied close to the long axis of the tooth. An intracoronal attachmenthowever, usually requires aboxpreparation to allow the attachment to fit within the crown contour. If it is not possible to createa box preparation that will totally incorporate the female element, then an extracoronal attachment should be considered. Since all intracoronal attachments are nonresilient, double abutting is recommended; ${ }^{4} 2$ ) extracoronal attachments, that positioned entirely outside the crown contour. Advantages of extracoronal attachments are that the normal tooth contour can be maintained, minimal tooth reduction is necessary and the possibility of devitalizing the tooth is reduced.Also, the path of insertion is easierfor patients with dexterity problems. Most extracoronal attachments have some type of resiliency (stress redirectors). Even with resilientattachment, double abutting is recommended whenever possible. It is, however, more difficult to maintain hygiene with extracoronal attachments and patients should be instructed on the use of dentalfloss and hygiene accessories. This will help prevent unnecessary tissue irritation caused by food entrapmentor calculus build-up;5,63) radicular and intraradicularstud type attachments thatare connected to a root preparation. The female or male is soldered or casted to a root cap coping. The female element of intraradicular stud type attachments fits within the root form contour. The SwissLogic, Zest and the ZAAG are examples of this type of attachment. Some stud type attachments, such as the UniAnchorand the DirectO-Ring, are directly cemented into the prepared root without requiring a cast coping. Stud type titanium implant attachments are 
also available to screw directly into implants or tissue extensions; ${ }^{4} 4$ ) bar type attachments span an edentulous area and connect abutment teeth, root or implant. The removable bridge, partial denture, or over denture fits over the bar and is connected to it with one or more retention sleeves, riders/ clips, or retentive plungers. ${ }^{4}$

Based on function, it is important to differentiate between a solidand resilient type restoration. Abutment/tooth supported restorations are considered solid, where abutment/tooth and tissue supported restorations are considered resilient. Abutment/tooth supported attachments are sub classified into two types, nonlockableand lockable. Resilient attachments are categorized into 5 classifications ranging from vertical to universal resiliency. The higher the number of classifications, the less torque transferred to the abutment, rootor implant. ${ }^{5}$

Based on modes of retention, they are frictional, mechanical, frictional \& mechanical, magnetic and suction types. Frictional retention is resistance to the relative motion of two or more surfaces in intimate contact with each other. Mechanical retention is resistance to the relative motion of two ormore surfaces due to a physical undercut. Magneticretention is the resistance to movement caused by a magnetic body that attracts certain materials by virtue of a surrounding field of force produced by the motion of its atomic electrons and the alignment of its atoms. Magnets do not provide lateral stability and are contraindicated for flatridges. It is used in limited applications, heat curing will weaken magnets and they are liable to corrode. Frictional and mechanical retention combines both features of frictional and mechanical retention as discussed. The score-PD attachment is a good example.Suction is a force created by a vacuum that causes a solidobjectadhere to a surface. An examplewould be a well-fitting denture. ${ }^{7}$

So, in this article is reported a rehabilitation of partially edentulous arch using semi precision attachment as an aesthetic approach

\section{CASE}

A 52-year-old female patient reported to the Department of Prosthodontics with a chief com- plaint of multiple missing teeth in maxillaryandmandibular arch, and inability to chew food properly and unaesthetic appearance. On intraoral examination, maxillary arch was completely edentulous and in mandibular arch 16, 17, 18, 24, 25, 26, 27 teeth were missing. All the teeth are still intact with good periodontal conditions (fig.1).

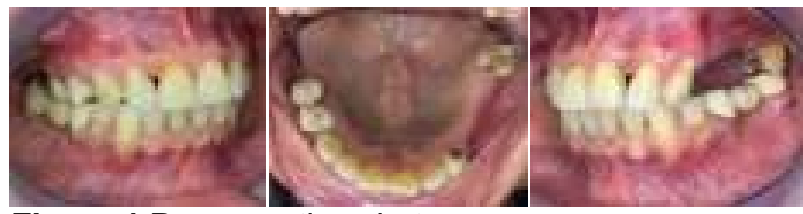

Figure 1 Pre-operative photos

\section{MANAGEMENT}

Patient was explained about various available treatment options like fixed partial denture combine with cast partial denture, overdenture and implant. Final treatment plan was chosen on considering the clinical conditions and patient'sesthetic need, functional requirementand economical condition. Maxillary arch was rehabilitated with splinting crown porcelain fused to metal on teeth 22-23, 14-15 which the Rhein $83^{8}$ extracoronal attachments were attached.

\section{Clinical procedure}

Impressions were made and the diagnostic casts were mounted on the articulator. Tooth preparation was done in $22,23,14$, and 15 for overdenture abutments. Measuring depth of preparation using putty index and the depth is $1.5 \mathrm{~mm}$ because the final restoration that we used is porcelain fused to metal (fig.2).

Final impression was made with lightbody elastomeric impression material and poured in die stone. The provisories crown was fabricated using putty indexand cemented using temporary acrylic (Charm-temp,Dentkist, Korea). Waxpatterns were fabricated for all the prepared teeth, and a wax custombar running overedentulous deficit ridge was connected to these prepared wax patterns. Ball attachmentpatterns (Rhein 83, USA) wereattached to the custom bar (fig.3). Selection criteria for precision attachment were based on location and length of the edentulous span.
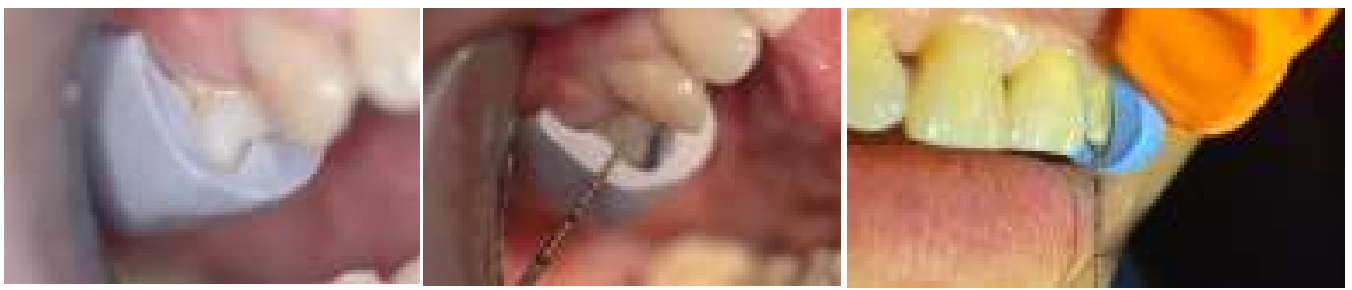

Figure 2 Measuring preparation using putty index) 


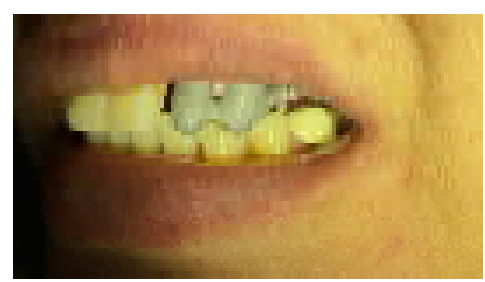

Figure 3 Try-in ball attachment (Rhein 83 USA)

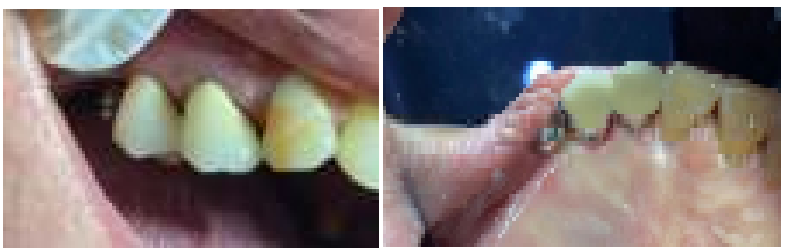

Figure 4 Try-in porcelain fused to metal crown

Aftertry-in, the next step was porcelain veneering on metal coping, then another try-in to check on PFMcrown margin on teeth and, alsocheck on the palatal side (fig.4).

Next impression used light body elastomeric impression material, with splint crownare attached to abutment. This impression was done to construct removable partial denture.

The waxocclusal rim was fabricated covering theedentulousarea. The jawrelation was recorded followed by articulation (fig.5) and teeth arrangement was done to achieve unilateral balanced occlusion with disclusion of all nonworking side teeth on lateral excursion. Waxed denture try-in was performed followed by acrylization with heat-polymerized acrylic resin (Trevalon HI, Dentsply, India). Laboratory remounting, finishing and polishing of the prosthesis were done. Standard retention caps were inserted in the slot present on the intaglio surface of the RPD.

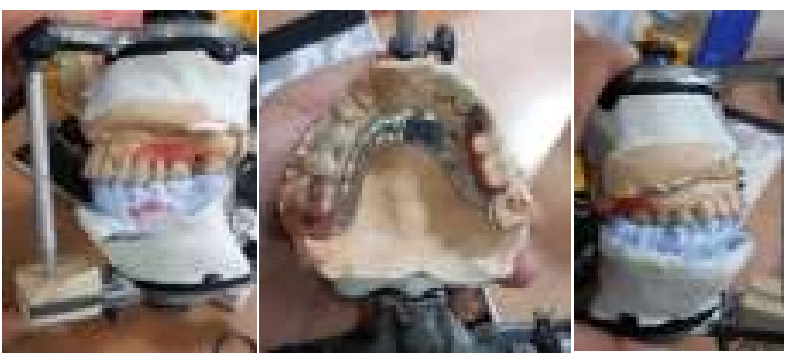

Figure 5 Jaw relation recorded on articulator

Cementation of a metal framework with auxiliary attachment was done using Type 1 Glass ionomer cement (GC Gold Label 1, Japan) and the removable denture was attached to this framework using the ball attachment. Postinsertion, hygiene, and home care instructions were explained to the patient. Recall visits of 1-and 3-month follow-up of the prosthesis were found to be satisfactory in terms of function and esthetics (fig.6).

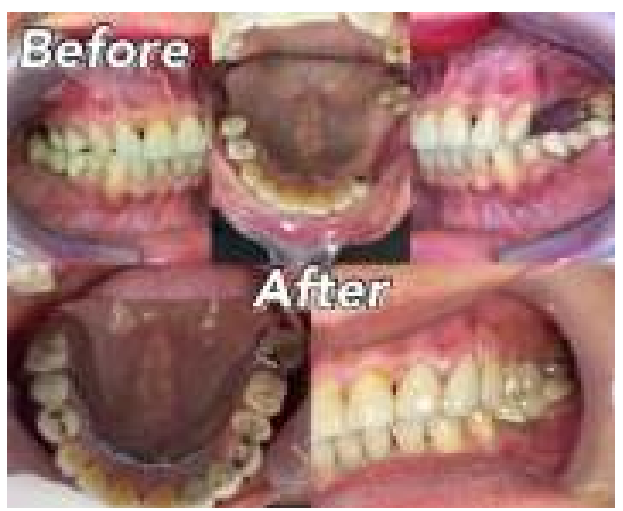

Figure 6 After insertion photos

\section{DISCUSSION}

In case of partially edentulous mouth, retention provided by the usage of precision attachments may have many advantages like comfort, chewing ability, as well as adequate distribution of occlusal loads and preservation of abutment teeth and less postoperativeadjustments in patients with removable partial denture. Precision attachments provide balance between functional stability and cosmetic appearance in partial denture. ${ }^{3}$

Conventional clasptype of removable partial denture is also a popular treatment choice as its lower cost, easy fabrication method and maintenance. But if patient demands for esthetics and abutment treatment of choice, precision attachment is an answer. ${ }^{2}$

The goals for the successful treatment in attachment retained cast partial denture include 2 main factors; developments of a stress directing attachment designand distribution of forces between the abutment teeth and residual ridge. ${ }^{4}$

This article describes rationale and technique for fabricating fixed-removable prosthesis using a precision attachment for rehabilitation of edentulous arch. Precision attachment retained RPD is a treatmentoption in unilateral orbilateral distal extension condition and if patient is more concerned about esthetic condition, but does not permit the use of dental implants, then precision attachment retained cast partial denture would be an excellent option as it provides adequate retention, stability, esthetics, and function.

\section{REFERENCES}

1. Prasad KD, Swaminathan AA. A Simplified approach to semi-precision attachment. Nitte Univ J Heal Sci 2020; 6:51-7.

2. Munot VK, Nayakar RP, Patil R. Case report prosthetic rehabilitation of mandibular defects with fixed-removable 
partial denture prosthesis using precision attachment: a twin case report. Contemp Clin Dent2017;8:11-9. doi: 10.4103/ccd.ccd

3. Pandey B, Joshi SP, Thapa D, Shrestha P. Use of a semi-precision attachment to fabricate a removable partial denture: a case report. J Nepal Prosthodont Soc 2019;2(2):108-12. doi:10.3126/jnprossoc.v2i2.31218

4. Patel B, Shah S, Sethuraman R, Chabra T, Gupta K. Rehabilitation of long span partially edentulous arch using claspless cast partial denture and precesion attachment-an aesthetic approach: case series. IOSR J Dent Med Sci 2019; 18:77-85. Doi:10.9790/0853-1801117785

5. Glossary of prosthodontic terms - 8. J Prosthet Dent 2005.

6. Preiskel HW. Precision attachment in prosthdontics. London: Quintessence Publishing Co Ltd; 1995

7. Preiskel HW. Precision attachments in prosthodontics: The applications of intracoronal and extracoronal attachments. Vol 1. Chicago: Quintessence Pub. Co.; 1984.

8. Rhein83USA. Attachments and pre-fabricated castable components: Catalog/technical manual for dentist and dental technicians: Available at: www.rhein83usa.com 